\title{
VARIAÇÃO E COMPETÊNCIA SOCIOLINGUÍSTICAS NO ENSINO DE INGLÊS COMO LÍNGUA ESTRANGEIRA
}

\author{
VARIACIÓN Y COMPETENCIA SOCIOLINGÜÍSTICA EN LA ENSEÑANZA DEL \\ INGLÉS COMO LENGUA EXTRANJERA
}

\author{
VARIATION AND SOCIOLINGUISTIC COMPETENCE IN ENGLISH AS A FOREIGN \\ LANGUAGE TEACHING
}

\author{
Juan Manuel HERNÁNDEZ-CAMPOY ${ }^{1}$ \\ Juan Antonio CUTILLAS-ESPINOSA ${ }^{2}$ \\ David BRITAIN ${ }^{3}$
}

RESUMO: Desde Currie (1952) usou o termo 'sociolinguística' e reivindicou seu status de disciplina autônoma, seu desenvolvimento e influência sobre outras áreas da pesquisa linguística nos últimos anos dentro e, principalmente, fora da Espanhade nossas fronteiras têm sido muito consideráveis. Um dos aspectos destacados pela Sociolinguística desde o início é a heterogeneidade social e linguística da linguagem. Portanto, se a variabilidade estrutural e regular é característica do uso normal da língua e também é a chave para entender os mecanismos de mudança lingüística, o conhecimento e o domínio dessa variação lingüística presente na comunidade também são muito importantes. intimamente ligado ao grau de competência sociolingüística do falante, de modo que todo aluno de ILE língua estrangeira deve estar ciente disso e, portanto, deve ser contemplado em materiais de ensino e de texto.

PALAVRAS-CHAVE: Variação linguística. Competência sociolinguística. Ensino ILE.

RESUMEN: Desde que Currie (1952) empleó el término 'sociolingüística' y reivindicó su estatus como disciplina autónoma, su desarrollo e influencia sobre otras esferas de la investigación lingüística durante los últimos años dentro y, principalmente, fuera de España, han sido muy considerables. Uno de los aspectos que la Sociolingüística ha destacado desde sus mismos albores es la heterogeneidad social y lingüística del lenguaje. Por ello, si la variabilidad estructural y regular es característica del uso normal de la lengua y constituye además la clave para la comprensión de los mecanismos del cambio lingüístico, el conocimiento y dominio de esta variación lingüística presente en el seno de la comunidad también está muy estrechamente ligado al grado de competencia sociolingüística del hablante, con lo que todo aprendiz de una lengua extranjera debe ser consciente de ella y, por ello, ha de ser contemplada en la docencia y los materiales de texto.

PALABRAS-CLAVE: Variación lingüística. Competencia sociolingüística. Enseñanza ILE.

\footnotetext{
${ }^{1}$ Universidade de Murcia (UM), Murcia - Espanha. Catedrático de Sociolinguística Inglesa. Licenciado em Filosofia e Letras (UM). ORCID: https://orcid.org/0000-0002-2282-7565. E-mail: jmcampoy@umu.es

2 Universidade de Murcia (UM), Murcia - Espanha. Professor Titular de Filologia Inglesa. ORCID: https://orcid.org/0000-0003-1478-4179. E-mail: jacuti@umu.es

${ }^{3}$ Universidade de Berna (UNIBE). Catedrático de Linguística Inglesa. ORCID: https://orcid.org/0000-00034228-0242. E-mail: david.britain@ens.unibe.ch
} 
ABSTRACT: Since Currie (1952) first used the term 'sociolinguistics' and claimed its status as an autonomous discipline, its development and influence over other areas of linguistic research in recent years have been very considerable within and, mainly, outside of Spain. The social and linguistic heterogeneity of language constitutes one of the most highlighted aspects in Sociolinguistics since its emergence as a discipline. Therefore, if regular and structural variability is characteristic of the normal use of the language as well as the key to understanding the mechanisms of linguistic change, similarly, the knowledge and command of this kind of linguistic variation present within the community is also very closely linked to the speaker's degree of sociolinguistic competence, so that every foreign language learner must be aware of its existence and, therefore, must be contemplated in EFL teaching and text materials.

KEYWORDS: Linguistic variation. Sociolinguistic competence. TEFL.

\section{Introdução: linguística aplicada, sociolinguística e línguas estrangeiras}

A pesquisa sobre o aprendizado e o ensino de línguas estrangeiras tem sido caracterizada desde o início pela adoção de perspectivas multidisciplinares e tratamento interdisciplinar, combinando com êxito a linguística com psicologia, pedagogia, educação, neurobiologia, sociologia ou antropologia (ver RICHARDS, 1974; VAN ELS et al., 1977; BELL, 1981; RUTHERFORD, 1984; ELLIS, 1985; 2012; STERN, 1983; DAVIES; CRIPER; HOWATT, 1984; COOK, 1993; RAMAT, 2002; ROBINSON; ELLIS, 2008; ELLIS; LOEWEN; ANCIÃO; ERLAM; PHILP; REINDERS, 2009; LASAGABASTER; DOIZ; SIERRA, 2014; LAMBELET; BERHELE, 2015; GABRY-BARKER; GA-AJDA, 2016; HABRAT, 2018; entre muitos outros). Essa multidisciplinaridade tem permitido importantes avanços na exploração dos processos de aprendizagem das línguas e no conhecimento de seu funcionamento, atores e fatores para poder aplicá-lo em seu ensino desde os trabalhos pioneiros em Linguística Aplicada como os de Lado (1957; 1964), Selinker (1972), Corder (1967; 1973) ou Krashen $(1981 ; 1982)$. De qualquer forma, a partir de suas várias propostas, houve um debate prolífico que ainda está longe do desenvolvimento de uma teoria globalizadora e integradora que tente explicar o aprendizado de línguas adicionais (L2/LE).

Muito tem sido escrito, por exemplo, uma vez que teorias contrastivistas mostraram na época que a interferência L1 poderia ser a fonte de erros cometidos pelo aprendiz de uma L2/LE, ou que estudos posteriores mostraram que muitos dos erros também fazem parte do desenvolvimento da própria L2 - como os cometidos por uma criança durante a aquisição de sua língua materna ou mesmo interlinguagem. Mas muitos outros não têm nada a ver com o L1 ou o L2, mas com fatores estritamente extralinguísticos de vários tipos. Diversos trabalhos têm destacado a importância da Sociolinguística, por exemplo, na aprendizagem e ensino de línguas 
estrangeiras ou segundas línguas (véanse SHUY, 1969; WHERRITT, 1981; JANICKI, 1982; WOLFSON; JUDD, 1983; PRESTON, 1989; GASS, 1989; BAYLEY; PRESTON, 1996; MCKAY; HORNBERGER, 1996; HERNÁNDEZ CAMPOY, 1997A; 1997B; SCHEU; HERNÁNDEZ-CAMPOY, 1998; PREISLER, 1999; HERNÁNDEZ CAMPOY; SCHEU, 2001; 2005; SEIDLHOFER, 2004; KIRKPATRICK, 2007; HORNBERGER; MCKAY, 2010; BAYYURT, 2013; GEESLIN; LONG， 2014; O BEAULIEU; WOLL; FRENCH; DUCHEMIN, 2018).

O conceito de "competência" inicialmente introduzido por Chomsky (1965) dizia respeito ao conhecimento linguístico geralmente inconsciente de uma pessoa sobre sua própria língua, ou seja, o sistema de regras que ele domina para ser capaz de produzir e entender uma série infinita de frases e reconhecer possíveis erros e ambiguidades gramaticais. Mas aprender a língua de uma determinada cultura não é apenas aprender muitas palavras nesse L2/LE, nem como construir uma infinita variedade de frases gramaticalmente corretas nesse L2/LE, mas aprender uma língua adicional também é aprender a conversar e interagir da mesma forma que um nativo sob as regras de interação comunicativa e valores típicos de seu sistema sociocultural. Assim, além da competência gramatical/linguística, surgiram a a sociocultural, a pragmática, discursiva, estratégica e comunicativa. Com essa motivação, e por analogia com o termo Chomskiano, introduziu Hymes (1966; 1972) o conceito de 'competição comunicativa': aprender uma língua não é apenas focar em dominar sua gramática, fonologia e vocabulário, mas também saber como usá-la adequadamente na sociedade em que é usada como nativa. $\mathrm{O}$ falante deve saber quando falar e quando não, que variedade e quando, quais fórmulas linguísticas usar, que estilo de acordo com a situação, e como ser cortês, rude, simpático ou hostil adequadamente. Portanto, a aquisição de competência comunicativa pode ser estabelecida nos mesmos termos da aquisição de competência gramatical. O que acontece é que tal competência comunicativa alimenta-se da própria experiência social e, portanto, precisa de mais tempo para ser adquirida (HYMES, 1972, p. 278).

Da mesma forma, se, durante o processo de aquisição de competência linguística na gramática da língua materna, as crianças também adquirem competência comunicativa, também, na aprendizagem de uma língua estrangeira, os alunos de língua, apesar de possuí-la em sua língua materna, têm que adquirir competência comunicativa, além da própria língua linguística, na língua estrangeira aprendem se quiserem usá-la de forma eficaz e apropriada para soar natural como se destaca en Wolfson e Judd (1983), Gass (1989), Scheu y HernándezCampoy (1998), Hernández-Campoy e Scheu (2001; 2005), Beaulieu, Woll, French, Leif y Duchemin (2018), entre outros. Isso supõe a aquisição dos valores culturais que fundamentam o 
discurso da comunidade L2/LE para usá-los adequadamente em qualquer situação e interpretar, com precisão, o que é dito, uma vez que as regras para o uso correto da fala mudam consideravelmente de uma sociedade para outra (WOLFSON; JUDD, 1983, p. 3-4). Esse contexto de língua em uso - de maior interesse em estudos de atuação do que competência - que a partir do final da década de 1960 começou a se propagar levou à aplicação de uma dimensão mais comunicativa da língua em sala de aula de língua estrangeira/segunda, tirando da gramática o lugar de alto privilégio que sempre teve quando se tratava de ensinar ou aprender uma língua (POULISSE, 1990, p. 6).

\section{Objetivos}

A Sociolinguística localizou e descreveu a simetria entre variação social e variação linguística em termos de variação sociolinguística, correlacionando fatores não linguísticos, como contexto situacional e parâmetros sociodemográficos com constituintes linguísticos. Mas se a variabilidade estrutural e regular é característica do uso normal da língua e também é a chave para entender os mecanismos de mudança linguística, o conhecimento e o domínio dessa variação linguística presente dentro da comunidade também está muito ligado ao grau de competência sociolingística do falante, com o qual cada aluno de uma língua estrangeira deve estar ciente e, portanto, deve ser coberto pelo ensino.

A competência sociolinguística é um componente integral da competência comunicativa que possui conhecimento pragmático e sociolinguístico para alcançar o uso linguístico e socialmente adequado (véanse MIZNE, 1997; YU, 2006; YA, 2010; PAULSTON; KIESLING; RANGEL, 2012; KRAMSCH, 2014; GEESLIN; LONG, 2014; O MEDE; DIKILITAS, 2015). Mas essa competência sociolinguística sempre foi baseada em uma competência linguística construída a partir de modelos de variedade padrão, tanto dialetal quanto de sotaque. Assim, embora um aprendiz de língua estrangeira tenha um grande domínio das regras da variedade não nativa nos diferentes espectros de competências (gramatical, sociocultural, pragmática, discursiva, estratégica ou comunicativa), sabendo como funcionar perfeitamente nas diferentes situações comunicativas praticadas naquela metalinguagem, esta ainda é a língua da sala de aula - e a variedade padrão nacional oficial - de modo que quando saem dessa bolha para o mundo real encontram uma variação sociolinguística e dialetal onde as variedades vernáculas não padrão predominam:

A primeira coisa que acontece a um aprendiz de inglês assim que chega às Ilhas Britânicas é que ele descobre (com grande doutment) o pouco que ele entende 
dos ingleses que ouve. Por um lado, porque as pessoas falam mais rápido do que o esperado. Por outro lado, porque os ingleses que falam mais britânicos são diferentes do que ele aprendeu. Além de ser surpreendido pelas diferenças de pronúncia, também notará diferenças na gramática e no vocabulário. (HUGHES; TRUDGILL, 1979, p. 1) $)^{4}$.

Portanto, como kramsch (2014) indica, "[...] nunca houve tanta discrepância entre o que é ensinado em sala de aula e o que os alunos precisarão no mundo real depois de saírem da sala de aula. Nas últimas décadas, esse mundo mudou a tal ponto que os professores de línguas não têm mais certeza do que devem ensinar ou as situações do mundo real para as quais devem preparar seus alunos" 5

O principal objetivo deste trabalho, portanto, é enfatizar que uma perspectiva sociolinguística que contemple a leitura socioeconômica e a variação dialetal, sensível à diversidade fora do padrão e destacando as grandes diferenças entre linguagem irreal e real, é indispensável para o ensino de línguas estrangeiras. Será exemplificado pelo caso da língua inglesa por ser a língua estrangeira mais amplamente ensinada e aprendida hoje.

\section{Variação sociolinguística e ensino de inglês como língua estrangeira}

Um dos aspectos que a sociolinguística tem insistido desde sua criação é o fato de que as línguas são entidades variáveis ao mesmo tempo que heterogênea social e linguisticamente. Assim, a Sociolinguística localizou e descreveu a simetria entre variação social e variação linguística em termos de variação sociolinguística utilizando fatores extralinguísticos sociodemográficos e de estilo. Mas se a variabilidade estrutural e regular é característica do uso habitual da língua e também é a chave para entender os mecanismos de mudança linguística, o conhecimento e o domínio dessa variação linguística presente dentro da comunidade também está muito ligado ao grau de competência sociolinguística do orador, com o qual cada aluno de uma língua estrangeira deve estar ciente dela e, portanto, deve ser contemplado no ensino, como destacaram Shuy (1969), Wherritt (1981), Preston (1989), Bayley y Preston (1996), Rickford (1996), Hernández-Campoy (1997a; 1997b), MompeánGonzález e Hernández-Campoy (2000); Kirkpatrick (2007), Hornberger e McKay (2010),

${ }^{4}$ Lo primero que le ocurre a un aprendiz de inglés en cuanto llega a las Islas Británicas es que descubre (con gran desencanto) lo poco que entiende del inglés que oye. Por una parte, porque la gente habla más rápidamente de lo esperado. Por otra, porque el inglés que habla la mayoría de los británicos es distinto del que él ha aprendido. Además de sorprenderle las diferencias de pronunciación, también percibirá diferencias en gramática y vocabulario. (HUGHES; TRUDGILL, 1979, p. 1, traducción nuestra).

5“"nunca ha habido tanta discrepancia entre lo que se enseña en el aula y lo que los estudiantes necesitarán en el mundo real una vez que hayan salido del aula. En las últimas décadas, ese mundo ha cambiado hasta tal punto que los profesores de idiomas ya no están seguros de lo que se supone que deben enseñar ni de las situaciones del mundo real para las que deben preparar a sus alumnos". 
Bayyurt (2013) ou Geeslin e Long (2014), e no contexto do inglês como língua franca (HARTMANN, 1996; GRADDOL; MEINHOF, 1999; MCKAY, 2010; PREISLER, 1999; GÖRLACH，2002； SEIDLHOFER，2004； MOMPEÁN-GONZÁLEZ; HERNÁNDEZCAMPOY, 2000; TONKIN; REAGAN, 2003; DZIUBALSKA-KOŁACZYK; PRZEDLACKA, 2008; ROSENHOUSE; KOWNER, 2008; ARCHIBALD; COGO; JENKINS, 2011; O DURHAM, 2014, entre outros).

\section{Variação de sotaque}

Todos nós falamos com um sotaque mais ou menos regional que pode revelar nossa origem geográfica e até social. Não seria difícil para nós, por exemplo, detectar, por exemplo, um castelhano, um andaluz, um murciano, um catalão, um galego ou um aragonês, através da pronúncia usada quando fala, embora envolvesse problemas de inteligibilidade para o estrangeiro aprender espanhol que não está familiarizado com esses sotaques. Da mesma forma, apesar da imagem tradicional transmitida em livros didáticos com um modelo e padrão homogêneo, as Ilhas Britânicas são caracterizadas por uma rica variedade de sotaques e dialetos regionais não padronizados, dos quais qualquer estudante de inglês como língua estrangeira deve estar pelo menos consciente para evitar a situação e as sensações do aprendiz descritas por Hughes y Trudgill $(1979$, p. 1). A dialetologia pode ser de grande utilidade para nós nesse sentido para nos fornecer dados em sala de aula sobretudo relacionado às principais variedades regionais do inglês britânico, diferente do padrão não só na pronúncia, mas também em detalhes gramaticais (vejam HUGHES; TRUDGILL, 1979; WELLS, 1982; TRUDGILL, 1990; TRUDGILL; CHAMBERS, 1991; CHESHIRE, 1991; MILROY; MILROY, 1993; FOULKES; DOCHERTY, 1999; SCHNEIDER; BURRIDGE; KORTMANN; MESTHRIE; UPTON, 2004; KORTMANN; BURRIDGE; MESTHRIE; SCHNEIDER; UPTON, 2004; KACHRU; KACHRU; NELSON, 2006; KIRKPATRICK, 2010; O BRITAIN, 2007A; 2010, entre outros). Da mesma forma, no mundo de língua inglesa, a pronúncia varia muito se olharmos para as características do sotaque dos estados americanos, canadenses, australianos, neozelandeses e sul-africanos, com suas respectivas variantes regionais internas, em comparação com as das Ilhas Britânicas (TRUDGILL; HANNAH, 1982; WELLS, 1982; CHESHIRE, 1991; SCHNEIDER; BURRIDGE; KORTMANN; MESTHRIE; UPTON, 2004; KACHRU; KACHRU; NELSON, 2006; O KIRKPATRICK, 2010). 
Para complicar ainda mais a situação, a Grã-Bretanha apresenta uma situação bastante peculiar como resultado da forte relação entre dialetos e sotaques, por um lado, e de origem social e regional, por outro. Assim, o RP é um sotaque não localizado, no sentido de que não revela nenhuma origem regional porque não pode estar associado a uma determinada região. No entanto, o RP revela formação social, e isso é importante para os estudantes de inglês: é a pronúncia que goza de um prestígio manifesto, uma vez que está associada a uma formação social e cultural muito específica que não são mais do que classes sociais com níveis econômicos suficientemente estáveis e altos, que lhes permitiram treinar nos centros onde a pronúncia de RP é ensinada (colégios particulares) vejam TRUDGILL, 1975; 1999; 2001; 2002; 2008; MOMPEÁN; HERNÁNDEZ-CAMPOY, 2000). Seguindo uma estrutura piramidal como a Trudgill (1975, p. 21) apontou, o inglês padrão é usado por $15 \%$ da população britânica, enquanto $85 \%$ dos britânicos são usuários de variedades não padronizadas. Por sua vez, o sotaque RP é falado por aproximadamente $5 \%$ dele, enquanto os 95\% restantes, a grande maioria, são usuários de sotaques regionais (ver Figura 1; TRUDGILL, 1975; 2001; 2002; 2008). Esta estrutura piramidal da contexto sociolinguístico britânica implica que quanto maior a posição social do falante, menos sotaque regional ele tem, e também menor o uso de formas léxicas e gramaticais locais.

Figura 4 - Pirâmide sociolinguística do inglês e suas variedades na Inglaterra

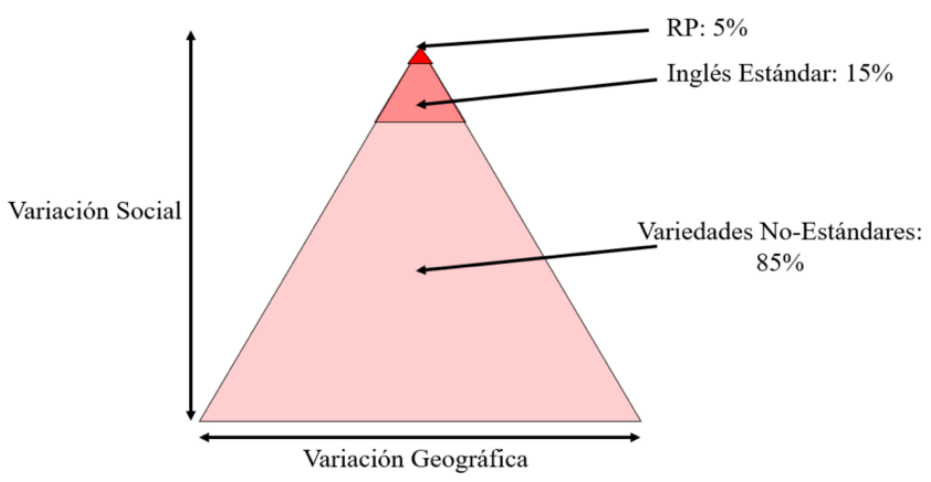

Fonte: Trudgill (1974)

No ensino de uma língua estrangeira, como é o caso do inglês, é necessário referir-se a essa situação característica da Grã-Bretanha, que não é dada em outros países ocidentais, como Alemanha, Estados Unidos ou Espanha, em que pessoas das mais altas e mais prestigiadas escalas sociais veem sua origem regional através da pronúncia, mesmo com os sotaques considerados prestigiados mais ligados a determinadas áreas geográficas do que a setores sociais específicos. Ensinar o sotaque RP tem suas vantagens e suas desvantagens (MOMPEÁN; HERNÁNDEZ-CAMPOY, 2000), embora o primeiro predomine, sem dúvida, 
mas todo aluno de inglês como língua estrangeira tem que estar ciente desse fato. Falar com o sotaque RP é uma grande vantagem, uma vez que o estudante que viaja para qualquer lugar nas Ilhas Britânicas nunca teria um problema de "ser compreendido" ao, por um lado, ser considerado lá com o sotaque inglês mais prestigiado, e, por outro lado, ser o mais conhecido entre a população britânica. Mas esse conhecimento só afeta a compreensão oral dos nativos, já que, embora todos entendam (lembre-se que é usado em todo rádio e televisão), nem todos o utilizam (5\%). Isso significa, portanto, que, do ponto de vista da compreensão oral do aluno, ele só entenderá facilmente esse $5 \%$ da população britânica, uma vez que os $95 \%$ restantes falam com sotaque local, o que é um inconveniente (TRUDGILL; HANNAH, 1982, p. 9). Além disso, outro dos problemas decorrentes de particular importância são os atitudinais, uma vez que um aspecto importante da complexa psicologia social das comunidades linguísticas é a resposta emocional dos membros da sociedade às linguagens e variedades em seu ambiente social. Na Grã-Bretanha, falantes com sotaques não padronizados são percebidos como menos inteligentes e menos educados, mas mais simpáticos, enquanto os usuários de sotaque de RP são vistos como mais inteligentes, mais educados, mas menos simpáticos (vide GILES, 1978; TRUDGILL, 1975).

Por outro lado, outro aspecto que a Sociolinguística tem insistido é que as comunidades linguísticas são heterogêneas tanto social quanto linguisticamente. Assim, uma de suas principais contribuições tem sido o conceito de variável sociolinguística para definir aqueles usos linguísticos que são formas alternativas de dizer o mesmo, embora sejam socialmente significativos, para denotar vários tipos de diferenciação social. Variáveis linguísticas do inglês britânico, como o (ng), (t) e (h) nas palavras, por exemplo, singing, butter, e hammer, respectivamente, tornaram-se variáveis sociolinguísticas nas comunidades de língua britânica onde são usadas: têm como variantes [y], [t] e [h] respectivamente no sotaque RP, enquanto em outros sotaques sua realização pode ser [n], [0], [0] ou $\varnothing$.Mas esses estudos também têm demonstrado que a variabilidade não é apenas uma questão de diferenças entre classes sociais e pronúncia, mas também de pronúncias diferentes dentro das mesmas classes sociais, dependendo das situações. Assim, nas Ilhas Britânicas, por exemplo, embora diferentes grupos de classes sociais tenham diferentes níveis de uso da variável (ng), sua avaliação das duas variantes é exatamente a mesma: falantes de todas as classes tendem a mudar sua pronúncia exatamente na mesma direção, elevando o percentual de uso da forma [y], pertencente ao RP de status social de prestígio, à medida que o nível de formalidade do contexto estilístico aumenta, e vice-versa. 


\section{Variação gramatical}

A sociologia da linguagem e da sociolinguística histórica pode ser de grande utilidade em nos deixar saber que, historicamente, o inglês padrão era - e de fato é - uma variedade dialeto semelhante a qualquer outra na Inglaterra, embora tenha sido estabelecido como o modelo (TRUDGILL, 1999) depois de ter sido modificado ao longo dos séculos pelo povo acadêmico (cortesãos, acadêmicos, escritores, etc.). É a variedade de inglês normalmente usada por pessoas cultas, usadas na imprensa e publicações, e a variedade geralmente ensinada em escolas britânicas para falantes nativos e não-nativos que aprenderão inglês como língua estrangeira. No entanto, seu uso não excede $15 \%$, como observado anteriormente, sendo a grande maioria (aproximadamente $85 \%$ ) usuários de variedades não padronizadas. Portanto, também é muito comum a estranheza demonstrada pelos estudantes de inglês ao ouvir ou ler declarações, geralmente encontradas no inglês mais comum - e até mesmo nas letras de canções de grupos anglo-americanos de pop e rock - que são construídas de forma muito diferente da que foram ensinadas através do inglês padrão em livros didáticos de referência e gramáticas (vide QUIRK; GREENBAUM; LEECH; SVARTVIK, 1985, por exemplo). Eles devem saber que o inglês padrão difere dos dialetos não padronizados em múltiplos aspectos gramaticais (vide WAKELIN, 1972; HUGHES; TRUDGILL, 1979; O’DONNELL; TODD, 1980; EDWARDS; TRUDGILL; WELTENS, 1984; TRUDGILL, 1990; TRUDGILL; CHAMBERS, 1991; CHESHIRE, 1991; MILROY; MILROY, 1993; KORTMANN; BURRIDGE; MESTHRIE; SCHNEIDER; UPTON, 2004; KACHRU; KACHRU; NELSON, 2006; KIRKPATRICK, 2010; O BRITAIN, 2007A; 2010, entre outros). No entanto, e infelizmente, na visão de Trudgill e Chambers (1991, p. 291), o estudo da variação das formas gramaticais presentes em dialetos não padronizados do Reino Unido tem sido consideravelmente menos abordado do que o relativo aos aspectos da pronúncia e do léxico, principalmente devido à dificuldade envolvida em seu tratamento e análise. Essa variação gramatical na língua inglesa afeta todos os níveis, desde sistemas pronominas e verbais, até formas morfossintáticas, como estruturas comparativas e superlativas, adverbiais e preposicionais (vide BRITAIN, 2007b; 2010).

Assim, por exemplo, se compararmos o sistema pronominal do inglês padrão com o de outras variedades dialetais, encontramos inúmeras diferenças, como a Tabela 1 resume (vide HUGHES; TRUDGILL, 1979; EDWARDS; TRUDGILL; WELTENS, 1984; TRUDGILL, 1990; 1999; 2003; O BRITAIN, 2007b; 2010). 
Tabela 1 - Sistema Pronominal do inglês padrão e variação regional

\begin{tabular}{|c|c|c|c|c|c|c|}
\hline \multicolumn{7}{|c|}{ Sistema Pronominal do inglês padrão e variação regional } \\
\hline Número & Pessoa & Pron-Sujeito & Pron.Objeto & Adj.Possessivo & Pron.Possessivo & $\begin{array}{l}\text { Pron.Reflexi } \\
\text { vo }\end{array}$ \\
\hline \multirow{5}{*}{ Singular } & $1^{\mathrm{a}}$ & I & $\begin{array}{l}\text { me } \\
\text { us }\end{array}$ & $\begin{array}{l}\text { my } \\
\text { me }\end{array}$ & mine & myself \\
\hline & $2^{\mathrm{a}}$ & $\begin{array}{l}\text { you } \\
\text { thou }\end{array}$ & $\begin{array}{l}\text { you } \\
\text { thee }\end{array}$ & your & $\begin{array}{l}\text { yours } \\
\text { yourn }\end{array}$ & yourself \\
\hline & $3^{\mathrm{a}}$ masc. & $\begin{array}{l}\text { he } \\
\text { him }\end{array}$ & $\begin{array}{l}\text { him } \\
\text { he }\end{array}$ & his & $\begin{array}{l}\text { his } \\
\text { hisn }\end{array}$ & $\begin{array}{l}\text { himself } \\
\text { hisself }\end{array}$ \\
\hline & $3^{\mathrm{a}}$ fem. & $\begin{array}{l}\text { she } \\
\text { her }\end{array}$ & $\begin{array}{l}\text { her } \\
\text { she }\end{array}$ & her & $\begin{array}{l}\text { hers } \\
\text { hern }\end{array}$ & herself \\
\hline & $3^{\mathrm{a}}$ neut. & It/that & it/that & its & its & itself \\
\hline \multirow{3}{*}{ Plural } & $1^{\mathrm{a}}$ & $\begin{array}{l}\text { we } \\
\text { us }\end{array}$ & $\begin{array}{l}\text { us } \\
\text { we }\end{array}$ & our & $\begin{array}{l}\text { ours } \\
\text { ourn }\end{array}$ & ourselves \\
\hline & $2^{\mathrm{a}}$ & $\begin{array}{l}\text { you } \\
\text { ye } \\
\text { youse }\end{array}$ & $\begin{array}{l}\text { you } \\
\text { youse }\end{array}$ & your & $\begin{array}{l}\text { yours } \\
\text { yourn }\end{array}$ & yourselves \\
\hline & $3^{\mathrm{a}}$ & $\begin{array}{l}\text { they } \\
\text { them }\end{array}$ & $\begin{array}{l}\text { them } \\
\text { they }\end{array}$ & their & $\begin{array}{l}\text { theirs } \\
\text { theirn }\end{array}$ & $\begin{array}{l}\text { themselves } \\
\text { theirselves }\end{array}$ \\
\hline
\end{tabular}

Fonte: elaboração própria

Os sistemas verbais também variam consideravelmente em toda a geografia do Reino Unido. Um fenômeno proeminente, por exemplo, é a ausência do final -s/es para a terceira pessoa singular dos verbos presentes na região de East Anglia, embora em vários dialetos do norte e sudoeste da Inglaterra e sul do País de Gales o fenômeno seja o contrário (Tabela 2)(Rupp y Britain 2019, por exemplo): 
Tabla 2 - Variação na marcação de $3^{a}$ pessoa do singular do presente em inglês britânico

Variação na marcação de $3^{\text {a }}$ pessoa do singular do presente em inglês britânico

\begin{tabular}{|l|l|l|}
\hline \multicolumn{1}{|c|}{ Inglês Padrão } & Dialetos do Norte e Sudoeste & \multicolumn{1}{c|}{ Dialeto de East Anglia } \\
\hline I take a cup of tea & I takes a cup of tea & Itake a cup of tea \\
You take a cup of tea & You takes a cup of tea & You take a cup of tea \\
He takes a cup of tea & He takes a cup of tea & He take a cup of tea \\
She takes a cup of tea & She takes a cup of tea & She take a cup of tea \\
We take a cup of tea & We takes a cup of tea & We take a cup of tea \\
You take a cup of tea & You takes a cup of tea & You take a cup of tea \\
They take a cup of tea & They takes a cup of tea & They take a cup of tea \\
\hline
\end{tabular}

Fonte: elaboração própria

Esse nivelamento apresentado por variedades não padronizadas baseia-se nas restrições do Northern Subject Rule e do Southern Subject Rule, que também afeta o nivelamento de was para todas as pessoas tanto do singular como do plural no pretérito do verbo to be. No primeiro caso, habitual nos dialetos norte e sudoeste, o nivelamento de was se dá quando o sujeito é um sintagma nominal (the cats was purring), ou um pronome sujeito não adjacente (the cats you brought was purring), enquanto que o Southern Subject Rule o nivelamento - $\emptyset$ se dá na condição inversa: quando o assunto é um pronome adjacente (they was purring). Nivelamento a were também ocorre para todas as pessoas, tanto singularmente quanto pluralmente, bem como nivelamento parcial: empregam was para todas as pessoas tanto no singular como plural em construções afirmativas mas, were com polaridade negativa (vide Tabela 3). A presença dos três padrões ocorre em diferentes e distantes geografias do mundo de língua inglesa (vide Rupp y Britain 2019). 
Tabla 3 - Nivelamento do pretérito do verbo TO BE

\begin{tabular}{|c|c|c|c|c|}
\hline \multicolumn{5}{|c|}{ Nivelamento do pretérito do verbo TO BE } \\
\hline \multirow{3}{*}{\begin{tabular}{|l} 
Raiz variável $s / \boldsymbol{r}$ \\
Inglês padrão \\
I was
\end{tabular}} & \multicolumn{4}{|c|}{ Regularización } \\
\hline & \multirow{2}{*}{$\begin{array}{l}\text { Nivelamento a WAS } \\
\text { I was }\end{array}$} & \multirow{2}{*}{$\begin{array}{l}\text { Nivelamento a WERE } \\
\text { I were }\end{array}$} & \multicolumn{2}{|c|}{ Nivelamento Misto } \\
\hline & & & I was & I weren't \\
\hline You were & You was & You were & You was & You weren't \\
\hline He/she/it was & He/she/it was & He/she/it were & He/she/it was & He/she/it weren't \\
\hline We were & We was & We were & We was & We weren't \\
\hline You were & You was & You were & You was & You weren't \\
\hline We were & We was & We were & We was & We weren't \\
\hline They were & They was & They were & They was & They weren't \\
\hline
\end{tabular}

Fonte: elaboração própria

A conjugação de verbos irregulares em inglês também está sujeita a variações consideráveis e até mesmo mudanças na regularização, adotando o sufixo dental -ed, como fazem os verbos regulares (draw-drew-drawn > draw-drawed-drawed). Em outros casos,a tendências é a redução das três formas a somente duas: do-did-done > do-done-done (vide HUGHES; TRUDGILL, 1979; TRUDGILL, 1990; 1999; 2003; O BRITAIN, 2007b; 2010):

Esses aspectos gramaticais não padronizados vistos, que são apenas uma amostra escassa, são os mais comuns de acordo com a pirâmide sociolinguística anteriormente oferecida, uma vez que são empregados por mais de $85 \%$ da população britânica, em comparação com $15 \%$ do uso da forma padrão de prestígio.

\section{Conclusão}

Portanto, assim como o estudo da língua estrangeira facilita a abordagem dos falantes e sua cultura, o conhecimento da cultura e da sociedade estrangeira nos permite aprofundar a compreensão da estrutura e do uso do LE. Precisamente, a ênfase na competência comunicativa no ensino de LEs, hoje, requer complementar o ensino com uma dimensão sociolinguística (vide DRESSLER; REUTER; REUTER, 1980; HAMMERLY, 1991; SCOLLON; SCOLLON, 1995; PAULSTON; KIESLING; RANGEL, 2012; O GEESLIN; LONG, 2014, entre muitos outros).

Por sua vez, se a variabilidade estrutural e regular é característica do uso normal da linguagem, o conhecimento e o domínio dessa variação dialética e socioeconômica presentes na comunidade também estão intimamente ligados ao grau de competência sociolinguística do 
falante. A incorporação dessa dimensão sociolinguística no ensino-aprendizagem de línguas estrangeiras ajudará a compreender melhor certas estruturas gramaticais e sequências fonológicas da língua-alvo e seu uso na sociedade, bem como estar ciente da diversidade e variação linguística existente nela, e permitirá reduzir o abismo que separa a meta linguística irrealista da sala de aula, a minoria e o vernáculo do mundo real, a maioria.

\section{REFERÊNCIAS}

ARCHIBALD, A.; COGO, A.; JENKINS, J. Latest trends in ELF research. Newcastle upon Tyne: Cambridge Scholars Press, 2011.

BAYLEY, R.; PRESTON, D. R. Second language acquisition and linguistic variation. Amsterdam: John Benjamins, 1996.

BAYYURT, Y. Current perspectives on sociolinguistics and english language education. The Journal of Language Teaching and Learning, v. 1, p. 69-78, 2013.

BEAULIEU, S.; WOLL, N.; FRENCH, L. M.; DUCHEMIN, M. Language learners' metasociolinguistic reflections: A window into developing sociolinguistic repertoires. System, v. 76, p. 210-218, 2018.

BELL, R.T. An introduction to applied linguistics. Approaches and methods in language teaching. Londres: Batsford Academic and Educational Ltd, 1981.

BRITAIN, D. Language in the British Isles. Cambridge: C.U.P., 2007a.

BRITAIN, D. Grammatical variation in England. In: BRITAIN, D. (Ed.) Language in the British Isles. Cambridge: Cambridge University Press, 2007b. p. 75-104.

BRITAIN, D. Grammatical variation in the contemporary spoken english of England. In: KIRKPATRICK, A. (Ed.). The Handbook of World Englishes. London: Routledge, 2010. p. 37-58.

CHAMBERS, J. K. Dynamic typology and vernacular universals. In: KORTMANN, B. (Ed.). Dialectology Meets Typology: Dialect Grammar from a Cross-linguistic Perspective. Berlín: De Gruyter, 2004. p. 127-145.

CHESHIRE, J. English around the world. Sociolinguistic perspectives. Cambridge: C.U.P., 1991.

CHOMSKY, N. Aspects of the theory of syntax. Cambridge, Mass.: MIT Press, 1965.

COOK, V. Linguistics and second language acquisition. Londres: Macmillan Education, 1993.

CORDER, S. P. The significance of learners' errors. International Review of Applied Linguistics in Language Teaching, v. 5, p. 161-170, 1967. 
CORDER, S. P. Introducing applied linguistics. Harmondsworth: Penguin Education, 1973.

CURRIE, H. A Projection of sociolinguistics: the relationship of speech to social status.

Southern Speech Journal, v. 18, p. 28-37, 1952.

DAVIES, A.; CRIPER, C.; HOWATT, A. P. R. Interlanguage. Edimburgo: Edinburgh University Press, 1984.

DURHAM, M. The acquisition of sociolinguistic competence in a lingua franca context. Bristol: Multilingual Matters, 2014.

DZIUBALSKA-KOŁACZYK, K.; PRZEDLACKA, J. English pronunciation models: a changing scene. Bern: Peter Lang, 2008.

EDWARDS, V. The grammar of southern british english. In: MILROY, J.; MILROY, L. (Eds.). Real English: the grammar of English dialects in the British Isles. London: Longman, 1993. p. 214-138.

EDWARDS, V.; TRUDGILL, P.; WELTENS, B. The grammar of english dialect: a survey of research. A Report to the ESRC Education and Human Development Committee. Londres: ESRC, 1984.

EISIKOVITS, E. Variation in the Lexical Verb in Inner-Sidney English. In: TRUDGILL, P. J.; CHAMBERS, J. K. (Eds.), 1991. p. 120-142.

ELLIS, R. Understanding second language acquisition. Oxford: O.U.P., 2015 [1985].

ELLIS, R. Language teaching research and language pedagogy. Malden: WileyBlackwell, 2012.

ELLIS, R; LOEWEN, S.; ELDER, C.; ERLAM, R.; PHILP, J.; REINDERS, H. Implicit and explicit knowledge in second language learning, testing and teaching. Bristol:

Multilingual Matters, 2009.

FOULKES, P.; DOCHERTY, G. Urban voices: accent studies in the british isles. Londres: Edward Arnold, 1999.

GABRYŚ-BARKER, D.; GAŁAJDA, D. Positive psychology perspectives on foreign language learning and teaching. Heidelberg: Springer, 2016.

GASS, S. Variation in second language acquisition: discourse and pragmatics. Bristol: Multilingual Matters, 1989.

GEESLIN, K.; LONG, A.Y. Sociolinguistics and second language acquisition: learning to use language in context. Londres: Routledge, 2014.

GILES, H. Patterns of evaluation in reactions to RP, south welsh and somerset accented speech. British Journal of Social and Clinical Psychology, v. 10, p. 280-281, 1971. 
GÖRLACH, M. English in Europe. Oxford: O.U.P., 2002.

GRADDOL, D; MEINHOF, U. H. English in a changing world. Special Issue of AILA Review 13, 1999.

HABRAT, A. The role of self-esteem in foreign language learning and teaching. Heidelberg: Springer, 2018.

HARTMANN, R. The english language in europe. Oxford: Intellect, 1996.

HERNÁNDEZ CAMPOY, J. M. Sociolingüística y enseñanza de lenguas extranjeras. In: OTAL, J. L.; FONTANET, I.; CODINA, V. (Eds). Estudios de Lingüística Aplicada. Castellón: Publicacions de la Universitat Jaume I, 1997a. p. 523-529.

HERNÁNDEZ CAMPOY, J. M. Sociolingüística y la Enseñanza del Inglés como Lengua Extranjera. In: AA.VV. (eds.). Estudios de Linguiística Aplicada y Literatura: Homenaje al Profesor Juan Conesa Sánchez. Murcia: EditUM, 1997b. p. 123-137.

HERNÁNDEZ CAMPOY, J. M.; SCHEU, D. La Competencia sociocultural en la enseñanza de lenguas extranjeras. Cuadernos de Filología Inglesa, v. 9, n. 2, p. 105-123, 2001.

HERNÁNDEZ CAMPOY, J. M.; SCHEU, D. La Importancia de la Competencia Sociocultural en el Contexto de la Enseñanza y Aprendizaje de Idiomas. In: AA.VV. (Eds.). Amica Verba: In Honorem Prof. Antonio Roldán Pérez. Murcia: Universidad de Murcia y CAM, 2005. p. 241-359.

HORNBERGER, N.; MCKAY, S. L. Sociolinguistics and language education. Bristol: Multilingual Matters, 2010.

HUGHES, A.; TRUDGILL, P. English accents and dialects: an introduction to social and regional varieties of english in the british isles. Londres: Edward Arnold, 1979.

HYMES, D. On Communicative Competence. In: PRIDE, J.; HOLMES, J. (Eds.). Sociolinguistics: Selected Readings. Harmondsworth: Penguin Books, 1972. p. 269-293.

JACNICKI, K. The foreigner's language in a sociolinguistic perspective. Poznan: Adam Mickiewicz University Press, 1982.

JENKINS, J. The phonology of english as an international language: new models, new norms, new goals. Oxford: O.U.P., 2000.

JENKINS, J. English as a lingua franca: attitude and identity. Oxford: O.U.P., 2007.

KACHRU, B. B.; KACHRU, Y.; NELSON, C. L. The handbook of world englishes. Oxford: Blackwell, 2006.

KIRKPATRICK, A. World englishes: implications for international communication and english language teaching. cambridge: C.U.P., 2007. 
KIRKPATRICK, A. The routledge handbook of world englishes. Londres: Routledge, 2010.

KORTMANN, B.; BURRIDGE, K.; MESTHRIE, R.; SCHNEIDER, E. W.; UPTON, C. A Handbook of varieties of english 2: morphology and syntax. Berlín: De Gruyter, 2004.

KRAMSCH, C. Teaching foreign languages in an era of globalization: Introduction. The Modern Language Journal, v. 98, p. 296-311, 2014.

KRASHEN, S. D. Second language acquisition and second language learning. Oxford: Pergamon, 1981.

KRASHEN, S. D. Principles and practice in second language acquisition. Oxford: Pergamon, 1982.

LADO, R. Linguistics across cultures: applied linguistics for language teachers. Ann Arbor: University of Michigan Press, 1957.

LADO, R. Language teaching: a scientific approach. Nueva York: McGraw-Hill

LAMBELET, A.; BERHELE, R. Age and foreign language learning. Londres: Palgrave MacMillan, 2015.

\section{LASAGABASTER, D.; DOIZ, A.; SIERRA, J. M. Motivation and foreign language} learning: from theory to practice. Amsterdam: John Bejamins, 2014.

MCKAY, S. English as an International Language. In: HORNBERGER, N.; MCKAY, S. L. (Eds.). Sociolinguistics and Language Education. Bristol: Multilingual Matters, 2010. p. 89-115.

MCKAY, S. L.; HORNBERGER, N. H. Sociolinguistics and language teaching. Cambridge: C.U.P., 1996.

MEDE, E.; DIKILITAŞ, K. Teaching and learning sociolinguistic competence: teachers' critical perceptions. Participatory Educational Research, v. 2, p. 14-31, 2015.

MILROY, J.; MILROY, L. Real english: the grammar of english dialects in the british isles. Londres: Longman, 1993.

MIZNE, C. A. Teaching sociolinguistic competence in the ESL classroom. 1997. Senior Thesis Projects, 1993-2002. Disponible en: http://trace.tennessee.edu/utk_interstp2/20. Acceso el: abr. 2020

MOELLER, A. K.; CATALANO, T. Foreign language teaching and learning. In: WRIGHT, J. D. (Ed.). International Encyclopedia for Social and Behavioral Sciences. Volume 9. 2. ed. Oxford: Pergamon Press, 2015. p. 327-332.

MOMPEÁN-GONZÁLEZ, J. A.; HERNÁNDEZ-CAMPOY, J. M. Advantages and Disadvantages of RP as an EFL Model of Pronunciation. In: DE LA CRUZ, I.; SANTAMARÍA, C.; TEJEDOR, C.; VALERO, C. (Eds.). La Lingüística Aplicada a 
Finales del Siglo XX. Ensayos y Propuestas. Alcalá: Universidad de Alcalá de Henares, 2000. p.707-713.

O’DONNELL, W. R.; TODD, L. Variety in contemporary english. Londres: Routledge, 1980.

PAULSTON, C. B.; KIESLING, S.; RANGEL, E. The handbook of intercultural discourse and communication. Oxford: Blackwell, 2012.

PREISLER, B. Functions and forms of English in a European EFL country. In: BEX, T.; WATTS, R. J. (Eds.). Standard English: The Widening Debate. Londres: Routledge, 1999. p. 239-268.

PRESTON, D. Sociolinguistics and second language acquisition. Oxford: Blackwell, 1989.

QUIRK, R.; GREENBAUM, S.; LEECH, G.; SVARTVIK, J. A comprehensive grammar of the english language. Londres: Longman, 1985.

RICHARDS, J.C. Error analysis: perspectives on second language acquisition. Londres: Longman, 1974.

RICKFORD, J. R. Regional and Social Variation. In: MCKAY, S. L.; HORNBERGER, N. H. (Eds.). Socio-linguistics and Language Teaching. Cambridge: England,1996. p. 151-194.

RAMAT, A. G. Typology and second language acquisition. Berlín: De Gruyter, 2002.

ROBINSON, P; ELLIS, N. Handbook of cognitive linguistics and second language acquisition. Londres: Routledge, 2008.

ROSENHOUSE, J.; KOWNER, R. Globally speaking: motives for adopting english vocabulary in other languages. Brisol: Multilingual Matters, 2008.

RUPP, L.; BRITAIN, D. Linguistic perspectives on a variable English morpheme: let's talk about -s. Londres: Palgrave, 2019.

RUTHERFORD, W. Language universals and second language acquisition. Amsterdam: John Benjamins, 1987 [1984].

SCHEU, U. D.; HERNÁNDEZ-CAMPOY, J. M. An analysis of sociocultural miscommunication: english, spanish and german. International Journal of Intercultural Relations, v. 22, n. 4, p. 375-394, 1998.

SCHNEIDER, E. W.; BURRIDGE, K.; KORTMANN, B.; MESTHRIE, R.; UPTON, C. A handbook of varieties of english 1: phonology. Berlín: De Gruyter, 2004.

SCOLLON, R.; SCOLLON, S. Intercultural communication. Oxford: Blackwell, 1995.

SEIDLHOFER, B. Research perspectives on teaching English as a lingua franca. Annual Review of Applied Linguistics, v. 24, p. 209-239, 2004. 
SELINKER, L. Interlanguage. International Review of Applied Linguistics, v. 10, p. 209241, 1972.

SHUY, R. The Relevance of Sociolinguistics for Language Teaching. TESOL Quarterly, v. 3, n, 1, p. 13-22, 1969

STERN, H. H. Fundamental concepts of language teaching. Oxford: O.U.P., 1983.

TONKIN, H.; REAGAN, T. Language in the $21^{\text {st }}$ Century. Amsterdam: John Benjamins, 2003.

TRUDGILL, P. Accent, dialect and the school. Londres: Edward Arnold, 1975.

TRUDGILL, P. The dialects of England. Oxford: Blackwell, 1990.

TRUDGILL, P. Standard English: What it isn't. In: BEX, T.; WATTS, R. (Eds.). Standard English: The Widening Debate. Londres: Routledge, 1999. p. 117-128.

TRUDGILL, P. Received Pronunciation: Sociolinguistic aspects. Studia Anglica Posnaniensia, v. 36, p. 3-13, 2001.

TRUDGILL, P. The sociolinguistics of modern RP. In: TRUDGILL, P. (Ed.) Sociolinguistic Variation and Change. Edimburgo: Edinburgh University Press. 2002. p. 171-180.

TRUDGILL, P. The historical sociolinguistics of elite accent change: on why RP is not disappearing. Studia Anglica Posnaniensia, v. 44, p. 1-12, 2008.

TRUDGILL, P.; CHAMBERS, J. K. Dialects of english: studies in grammatical variation. Nueva York: Longman, 1991.

VAN ELS, T.; BONGAERTS, T.; EXTRA, G.; VAN OS, C.; JANSEN-VAN DIETEN, A. Applied linguistics and the learning and teaching of foreign languages. Londres: Edward Arnold, 1984 [1977].

WHERRITT, I. Sociolinguistics and the teaching of foreign languages. System, v. 9, n. 2, p. 141-150, 1981.

WOLFSON, N.; JUDD, E. Sociolinguistics and language acquisition. Massachusetts: Newbury House Publishers, 1983.

YA, L. I. U. A study of language teaching from a sociolinguistic perspective of communicative competence. Canadian Social Science, v. 4, n. 3, p. 80-86, 2010.

$\mathrm{Yu}, \mathrm{M}$. C. On the teaching and learning of L2 sociolinguistic competence in classroom settings. Asian EFL Journal, v. 8, n. 2, p. 1-24, 2006. 


\section{Como referenciar este artigo}

HERNÁNDEZ-CAMPOY, Juan Manuel; CUTILLAS-ESPINOSA, Juan Antonio; BRITAIN, David. Variação e competência sociolinguísticas no ensino de inglês como língua estrangeira. Rev. EntreLínguas, Araraquara, v. 6, n. 1, p. 183-201, jan./jun., 2020. e-ISSN: 2447-3529. DOI: https://doi.org/10.29051/el.v6i1.13379

Submetido em: 30/07/2019

Revisões requeridas em: 30/08/2019

Aceito em: 30/11/2019

Publicado em: 06/01/2020 